\title{
Inspiration-induced vasoconstrictive responses in dominant versus non-dominant hands
}

\author{
Harvey N. Mayrovitz and Edye E. Groseclose \\ College of Medical Sciences, Nova Southeastern University, Ft Lauderdale, FL, USA
}

\section{Summary}

\section{Correspondence}

Dr Harvey N. Mayrovitz, College of Medical Sciences, Nova Southeastern University, 3200 S.

University Drive, Ft. Lauderdale, FL 33328, USA

E-mail: mayrovit@nova.edu

\section{Accepted for publication \\ Received 21 August 2004; \\ accepted 8 October 2004}

\section{Key words}

finger; hand; inspiratory gasp; laser Doppler; microcirculation; reflexes neurovascular; skin blood flow; vascular control
Single rapid and deep inspirations (inspiratory gasps, IG) result in arteriolar vasoconstriction with concomitant transient decreases in skin blood flow that are most prominent in fingers and toes. Vascular responses (inspiratory gasp responses, IGR) are determined as the maximum percentage reduction in blood flow and have been used to assess sympathetic neurovascular function in several conditions. Previous studies have described various features of the response but there has been no reported systematic investigation of the degree of similarity between IGR obtained on dominant and non-dominant hands. This aspect is important in procedures that may use IGR to evaluate suspected unilateral sympathetic dysfunction of a limb-pair or to test the effectiveness of physiological interventions imposed on a single limb of a pair. Thus, the goal of our study was to compare IGR magnitudes that were simultaneously determined in paired-fingers of dominant and non-dominant hands. In 30 healthy seated subjects, skin blood perfusion via laserDoppler (SBF) was measured on the dorsum of the middle finger of both hands while subjects performed three sequential IG at 3-min intervals. Analysis of variance for repeated measures revealed no significant difference in IGR between dominant $(79.3 \pm 11 \cdot 2 \%)$ and non-dominant hands $(81.9 \pm 11.6 \%, P=0.965)$ with an overall IGR of $80.6 \pm 11.4 \%$. These results indicate that hand-dominance is not a factor that is likely to significantly effect IGR differentials determined in pairedlimbs.

\section{Introduction}

The inspiratory gasp response (IGR) is a vasomotor reflex triggered by a rapid and deep inspiration that results in an arteriolar vasoconstriction and a concomitant transient decrease in skin blood flow. This response, first identified by Bolton and co-workers in 1936 (Bolton et al., 1936), is most prominent in fingers and toes, and has been used as a quantitative measure of sympathetic neurovascular function in a variety of conditions (Netten et al., 1996; Birklein et al., 1998; Hilz et al., 1999; Littleford et al., 1999). One potential limitation of the IGR in both experimental and clinical settings is its temporal and subject-by-subject variability (Mayrovitz \& Groseclose, $2002 a$,b). Factors that may contribute to the variability include the point in the respiratory cycle at which the inspiration is initiated (Du Buf-Vereijken et al., 1997), the elapsed time between consecutive inspirations (Mueck-Weymann \& Rauh, 2002; Rauh et al., 2003), local skin temperature and resting blood flow (Oberle et al., 1988; Khan et al., 1991), subject age
(Khan et al., 1992; Lau et al., 1995) and possibly the subject's vital capacity (Liu et al., 1996). Limitations imposed by some components of subject-to-subject variability might be lessened under conditions in which comparisons of IGR between pairedlimbs are suitable for a particular experimental design or clinical study. This would be applicable to experiments designed to evaluate suspected unilateral sympathetic dysfunction of a limbpair or to test the effectiveness of treatments or physiological interventions imposed on a single limb of a pair. The utility of paired-limb comparisons, in the case of IGR determined in fingers, would be expected to be influenced by the degree of similarity of dominant and non-dominant hand responses. Although there is some data regarding single IGR determined sequentially in left and right hands (Schurmann et al., 1996), systematic comparisons between dominant versus non-dominant hand responses has not been reported. Thus, this study was undertaken with the focused goal of comparing the magnitudes of IGR, measured simultaneously in paired-fingers of the dominant and non-dominant hands of healthy subjects. 


\section{Methods}

\section{Subjects}

Healthy young subjects $(n=30$, age $27 \cdot 9 \pm 1 \cdot 1$ years, 15 male $)$ participated after signing a consent form approved by the university institutional review board. Subjects had no history of vascular disease, did not have diabetes and were not taking any form of vasoactive medication. Overall, the subjects' parameters (mean \pm SEM) were: height $172.2 \pm 3.9 \mathrm{~cm})$, weight $(72.8 \pm$ $5.5 \mathrm{~kg})$ and systolic $(114 \pm 4)$ and diastolic $(75 \pm 2)$ blood pressures $(\mathrm{mmHg})$. Although not a requirement, the right hand was dominant for all subjects.

\section{Skin blood perfusion}

Skin blood perfusion (SBF) was measured using a dual-channel laser-Doppler flowmetry system (Moor Instruments model MBF3D, Moor Instruments, Inc. Wilmington, Delaware USA) with probes (model DP7a) affixed to the dorsal surface of the each middle finger with paper tape (Fig. 1). These are integrating probes, with eight light collecting fibres surrounding a central fibre bundle that carries the incident laser light. Flow outputs of the monitoring system underwent A/D conversion (DataQ model 720B, DATAQ Instruments, Inc. Akron, Ohio USA) at a sampling rate of 100 samples s$^{-1}$ and were recorded at a standardized gain on a dedicated computer. Each probe was calibrated in its associated channel using a motility standard supplied by the manufacturer. In addition, prior to each experiment, the outputs of each probe-channel combination were tested using a rotating disk (2 revolutions $\mathrm{min}^{-1}$ ) with imbedded, randomly arranged particles, to simulate moving cells. The probe-channel system output was monitored for $3 \mathrm{~min}$ and its mean output averaged. If the mean outputs between channels differed by more $4 \%$ the system was recalibrated.

Principles of laser-Doppler perfusion measurements have been previously published (Nilsson et al., 1980; Oberg et al., 1984; Mayrovitz, 1998). Briefly, a low intensity laser light signal is transmitted into the skin to a depth of about 1-2 mm and the reflected light is used to measure local blood perfusion. The Doppler-shifted signal contains information about the speed and number density of moving red blood cells in a tissue region to a depth of about 1-2 mm (Jakobsson \& Nilsson, 1993). Speed and number density information is processed to yield a parameter, perfusion, that is proportional to blood flow and usually expressed in relative perfusion units (rpu). Probes were directly attached to the skin with tape and received blood perfusion data from a surface area of about $8 \mathrm{~mm}^{2}$ which was transmitted via a fibre optic bundle to the central processor. The frequency cut-off was set to $14 \cdot 7 \mathrm{KHz}$ and the response time was $0 \cdot 1 \mathrm{~s}$ for all experiments.

An intrinsic feature of laser-Doppler perfusion measurements is the presence of a small non-zero signal known as the ‘biological zero' (BZ) (Abbot \& Beck, 1993; Kernick et al., 1999; Mayrovitz \& Leedham, 2001). It is standard practice to determine BZ using a vascular occlusion to reduce the flow to zero and then subtract the BZ value so determined from all recorded perfusion values. This was done in the present study at

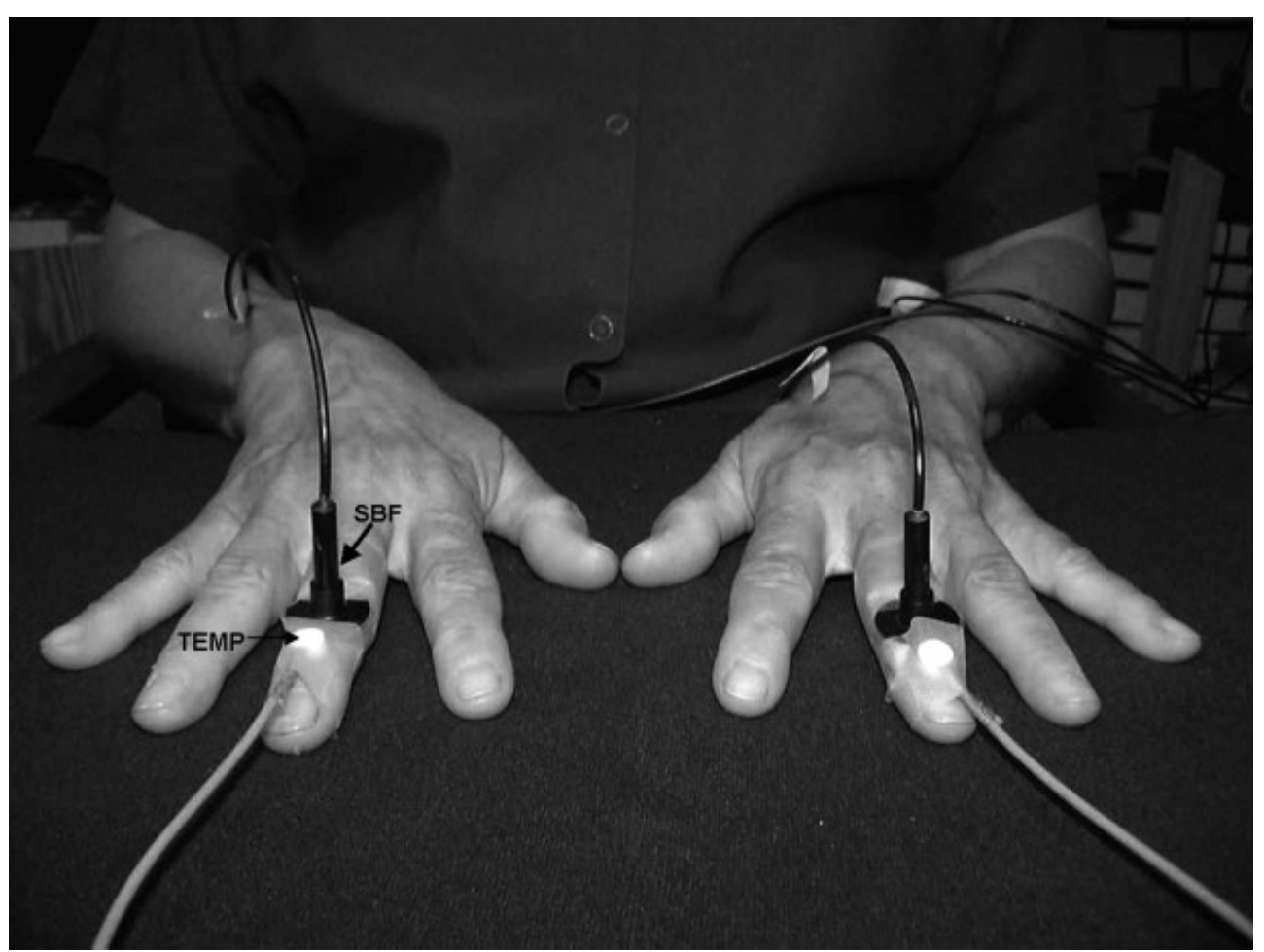

Figure 1 Hand position and measurements during the experimental procedure. Laser Doppler probe (SBF) measures finger skin blood perfusion and thermocouple (TEMP) measures temperature. 
the end of each protocol using a vascular occluder-cuff placed around the base of each middle finger. Occlusions were made simultaneously using two sphygmomanometers to which the occluder-cuffs were connected. The duration of occlusion was 2 min, which is ample to determine BZ (the lowest value recorded during the zero-flow occlusion interval). The BZ correction for the present study was small in relation to the resting average flows measured for all subjects (3·15 $\pm 0 \cdot 24 \%)$.

\section{Temperatures}

Skin temperature was monitored continuously with a thermocouple placed on each finger dorsum slightly distal to the laserDoppler probe (Fig. 1). Each thermocouple was connected to a monitor (Physitemp, Model TH-8, Physitemp Instruments, Inc. Clifton, New Jersey USA). Monitor outputs were recorded on separate channels of the data acquisition computer. Room temperature was recorded at the beginning and end of the protocol. For the entire group of subjects, initial room temperature ranged from $23.9^{\circ}$ to $25 \cdot 1^{\circ} \mathrm{C}$, but for any given subject, room temperature variation was $<0.5^{\circ} \mathrm{C}$ from start to finish. Room relative humidity for the entire group varied from 47 to $59 \%$, but variation during any given subject's full protocol was $<2 \cdot 1 \%$. For the entire group, skin temperature of left hands (mean $\pm \mathrm{SD}$ ) was $33.8 \pm 1 \cdot 1{ }^{\circ} \mathrm{C}$ which was not significantly different from that measured on right hands $\left(33.7 \pm 1 \cdot 2{ }^{\circ} \mathrm{C}\right.$, $\mathrm{P}=0 \cdot 709)$.

\section{Protocol}

During a set-up interval, subjects sat quietly with their hands placed comfortably, palm down, on a supporting surface that was attached to the arms of their chair. Laser-Doppler and thermocouple probes were applied bilaterally to the middle finger dorsum. During the next 10 min subjects were instructed as to the breathing manoeuvre that was required and were given at least two chances to practice. The instruction was to take a deep and rapid inspiration starting at the end of a normal quiet expiration. Subjects were instructed to first sense their breathing pattern so that they could feel comfortable in identifying the endexpiratory point and then to take the gasp when comfortable. During testing, the experimenter quietly informed the subject that their next gasp was in $15 \mathrm{~s}$ and, when the time arrived, they were instructed to 'gasp when ready'. Following this last instruction, subjects usually initiated the gasp within $10 \mathrm{~s}$. After set-up and instruction, data recording was started and baseline average perfusion was recorded for $10 \mathrm{~min}$. At $10 \mathrm{~min}$ into the recording, the first gasp was initiated. The initial gasp was followed $3 \mathrm{~min}$ later by a second gasp, then $3 \mathrm{~min}$ later by a third (Fig. 2). Biological zeros and blood pressures were measured at the end of the experimental sequence.

\section{Data reduction}

\section{Resting skin blood perfusion}

The average SBF during the $10 \mathrm{~min}$ baseline interval that preceded the IGR experimental sequence was determined for each instrumented finger and used to characterize the resting SBF for each subject.

\section{Inspiratory gasp responses}

The average SBF during the 20-s interval immediately preceding each successive gasp was used as a reference perfusion for its following inspiratory gasp vascular response. IGR was determined from the minimum perfusion during the gasp ( $\left.\mathrm{SBF}_{\mathrm{min}}\right)$ and its reference perfusion $\left(\mathrm{SBF}_{0}\right)$ according to the relationship:

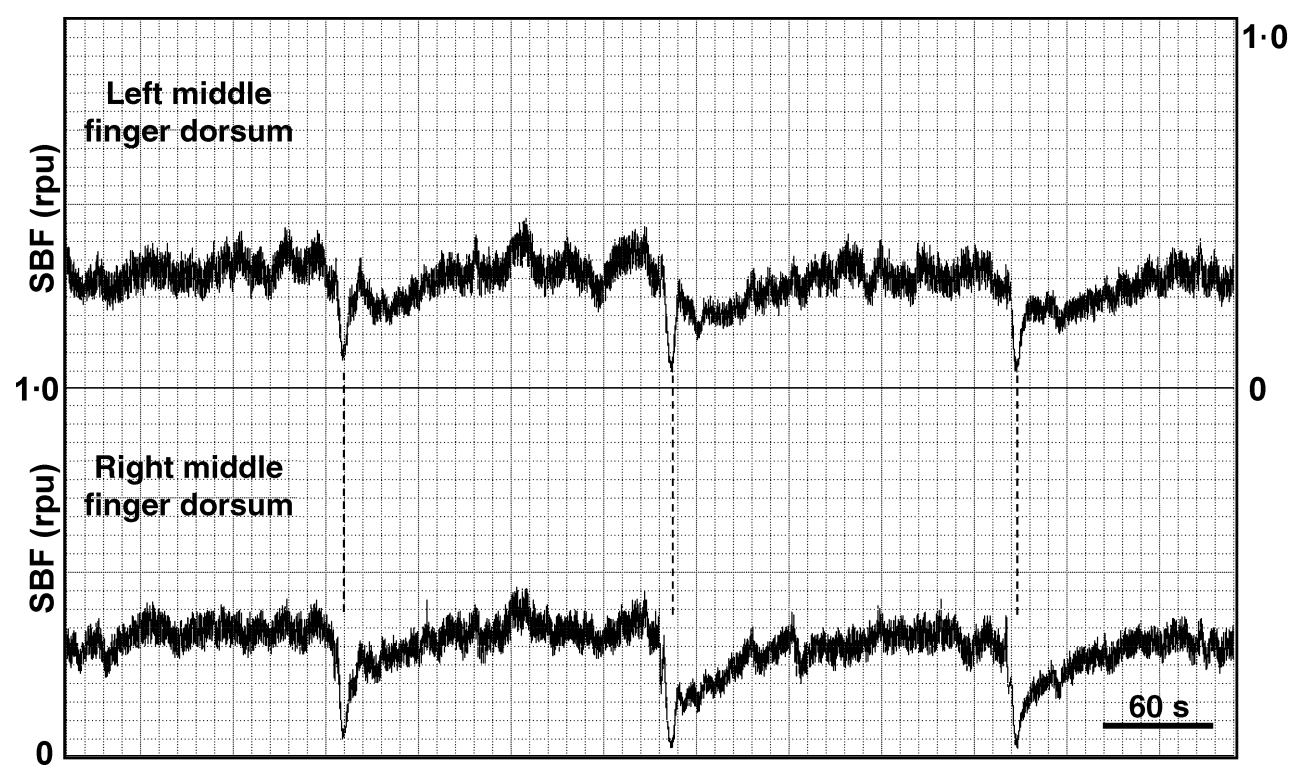

Figure 2 Sequential procedure for triplicate inspiratory gasps. Skin blood perfusion (SBF) is monitored continuously and three consecutive inspiratory gasps are taken at intervals of $180 \mathrm{~s}$. These occur at the vertical dashed lines where SBF in both fingers is seen to decrease. 


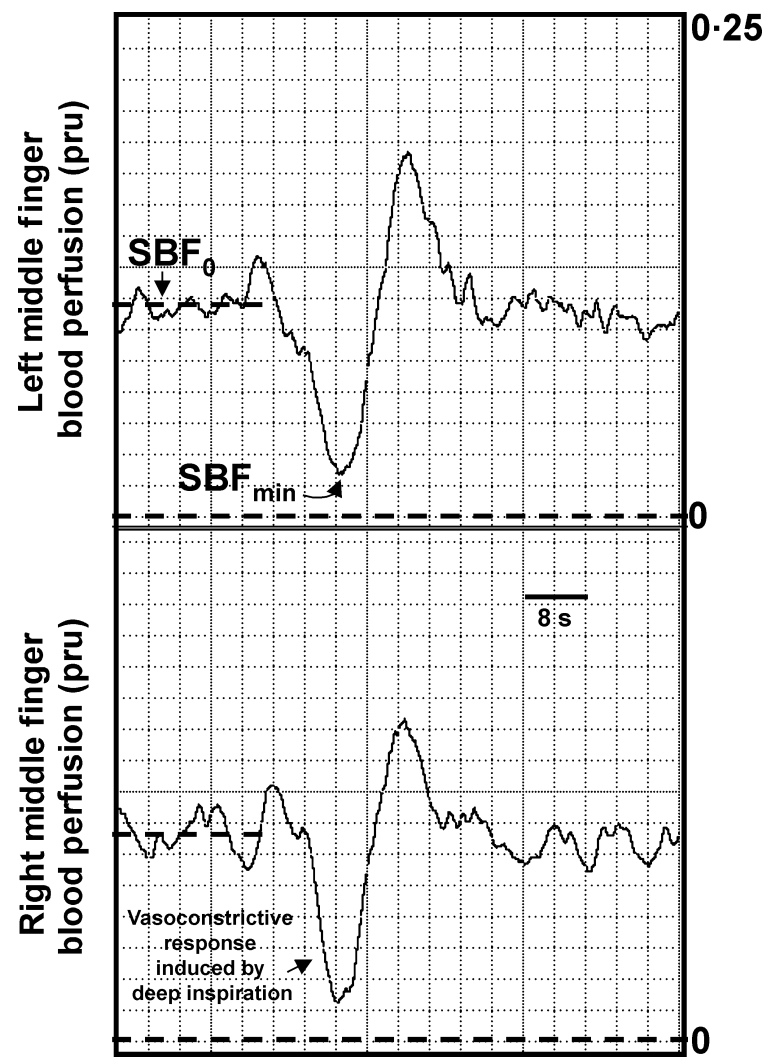

Figure 3 Determination of the inspiratory gasp response (IGR). A deep inspiration causes a vasoconstriction and a transient decrease in measured laser-Doppler blood perfusion ( $\mathrm{SBF}) . \mathrm{SBF}_{0}$ is the mean perfusion during the immediately preceding 20 -s interval and $\mathrm{SBF}_{\min }$ is the minimum perfusion achieved during the response. IGR is calculated by the expression IGR $=100 \times\left(\mathrm{SBF}_{0}-\mathrm{SBF}_{\min }\right) / \mathrm{SBF}_{0}$. Vertical axis is perfusion in relative perfusion units (rpu) with same scale for upper and lower panels.

IGR $=100 \times\left(\mathrm{SBF}_{0}-\mathrm{SBF}_{\min }\right) / \mathrm{SBF}_{0}$ (Fig. 3). Accordingly the maximum range of IGR was $0-100 \%$.

\section{Analysis}

The primary data set consisted of three sequential IGR obtained simultaneously for each hand of 30 subjects (Fig. 3). All statistical analyses were performed using the Statistical Package for the Social Sciences (SPSS) software (version 7·0). The data were evaluated for ANOVA suitability with respect to normality of distribution and homogeneity of variances; no data were omitted from analysis. To determine whether there was a significant difference in IGR $(\mathrm{P}<0.05)$ between dominant (right) and non-dominant (left) hands, a general linear model for repeated measures was used with hand and gender as factors. Tests for differences in resting SBF between hands and genders were done using ANOVA.

\section{Results}

Values quoted are mean and SD. SBF is expressed in pru and IGR in per cent.
Table 1 Sequential IGR.

\begin{tabular}{lccc}
\hline & First IGR & Second IGR & Third IGR \\
\hline Left-hand IGR (\%) & $84 \cdot 2 \pm 14 \cdot 1$ & $79 \cdot 8 \pm 13 \cdot 0$ & $81 \cdot 9 \pm 14 \cdot 9$ \\
Right-hand IGR (\%) & $80 \cdot 9 \pm 11 \cdot 9$ & $77 \cdot 4 \pm 13 \cdot 9$ & $79 \cdot 6 \pm 14 \cdot 1$ \\
Maximum difference (\%) & $9 \cdot 6 \pm 8 \cdot 5$ & $10 \cdot 6 \pm 9 \cdot 1$ & $10 \cdot 5 \pm 7 \cdot 9$ \\
\hline
\end{tabular}

Values are mean $\pm \mathrm{SD}$. Maximum difference is absolute value of difference between hands.

\section{Resting skin blood perfusion}

Analysis of variance showed that resting SBF for left $(0 \cdot 238 \pm 0 \cdot 146)$ and right $(0 \cdot 224 \pm 0 \cdot 112)$ hands were similar in value and not significantly different from each other $(\mathrm{P}=$ $0 \cdot 759)$. There was also no significant difference in SBF detected between females $(0 \cdot 226 \pm 0 \cdot 136)$ and males $(0 \cdot 239 \pm 0 \cdot 139)$ $P=0.694$.

\section{Inspiratory gasp responses}

In comparing IGR, the general linear model analysis for repeated measures revealed neither significant difference in IGR between right hands $(79 \cdot 3 \pm 11 \cdot 2)$ and left hands $(81 \cdot 9 \pm 11 \cdot 6, \mathrm{P}=$ $0 \cdot 965)$, nor a significant difference in IGR between genders $(P=0 \cdot 216)$, although IGR in males $(83.4 \pm 10 \cdot 6)$ tended to be larger than for females $(78 \cdot 7 \pm 11 \cdot 7)$. The overall IGR for all hands was $80 \cdot 6 \pm 11 \cdot 4$. Table 1 summarizes absolute IGR obtained for first, second and third IGR pairs and also the maximum difference in IGR between hands determined as the absolute difference between hands for each of the sequential IGR. There was no significant difference among sequential IGR for either the left hand $(\mathrm{P}=0.422)$ or the right hand $(\mathrm{P}=$ $0.501)$ nor was the absolute difference in IGR between hands significantly different $(P=0 \cdot 470)$. These results suggest the absence of any habituation effect due to the repetitive IGR.

\section{Discussion}

The main goal of this study was to compare bilateral IGR that were simultaneously induced and measured on the finger dorsum of dominant (right) and non-dominant (left) hands. Analysis of bilateral triplicate IGR revealed no significant difference between right and left hand IGR in this normal population of young healthy subjects. At a power of $0 \cdot 8$, the smallest effect size (paired-limb difference/SD) that this study could detect was $0 \cdot 75$. Based on the overall SD of $\pm 11 \cdot 4$, this effect size estimates the difference between hands to be less than $\pm 10.6 \%$ with either hand having an IGR in the interval $72 \cdot 0-89 \cdot 1$. These findings strictly apply to the finger dorsum of right-handed persons.

The overall IGR of $80.6 \pm 11.4$ of the present study is somewhat larger in magnitude and less variable than previously found values $(72 \cdot 2 \pm 16 \cdot 7)$ in a group of 28 healthy subjects of similar age (Mayrovitz \& Groseclose, 2002b), Although the 
mean value previously obtained falls within the estimated confidence interval of the present study, the larger SD is not so easily explained. We suspect that part of the source of the larger variability previously reported was due to the use of a single fibre laser Doppler probe as compared with the multi-fibre integrating probe used here. The multi-fibre probe tends to smooth out spatial variability resulting in less overall variability in perfusion values. We also suspect that the previous way of determining the reference perfusion $\left(\mathrm{SBF}_{0}\right)$ for each IGR also contributed to the larger variability. Previously $\mathrm{SBF}_{0}$ was determined as the average SBF during the $120 \mathrm{~s}$ immediately preceding each of the sequential IGR. Under these conditions $\mathrm{SBF}_{0}$ may have variably included elevated or reduced flow values if flow was still recovering from the immediately preceding IGR. In the present study $\mathrm{SBF}_{0}$ was determined over a $20 \mathrm{~s}$ interval immediately preceding each IGR. This would be expected to reduce potential variability induced by flow changes related to the immediately previous IGR.

The only other study known to us in which left and right hand responses were compared (Schurmann et al., 1996), did so using single IGR obtained first on one hand and then on the other, with the site of measurement being the palmar finger tip heated to $40^{\circ} \mathrm{C}$. These authors also found no significant difference between hands, with left versus right IGR being $66 \pm 17$ versus $69 \pm 13$. Based on their measurements in 52 subjects, with ages ranging from 17 to 75 years, they defined a normal physiological range as the mean $\pm 2 \mathrm{SD}$, which for their data gave an IGR range of 37-97. Application of the 2 SD criterion to the present data yields an IGR range of $57 \cdot 8-100$. The lower mean response value and greater SD they report may reflect the greater age range and older average age of their combined group $(40 \cdot 1$ years) as compared with the present group (27.9 years). Age-related reductions in IGR have previously been reported (Khan et al., 1992). Other factors that make these two studies not directly comparable include the finger measurement site (palmar versus dorsum), the subject's posture (supine versus seated) and the local skin temperature (heated to $40^{\circ} \mathrm{C}$ versus non-heated).

Both palmar and dorsal aspects of fingers are suitable to demonstrate the IGR. We chose to use the dorsal site mainly because it was technically easier and mechanically more reliable to place the laser-Doppler probes vertically on the dorsum rather than downward from the finger pulp. The choice of the middle finger versus the index finger was made because in most people the distal surface of the middle finger is flatter and has a greater surface area making probe placement and maintenance more stable. It is also true that the dorsal skin region tends to have less arterial-venous shunts that function exclusively in thermal regulation. Because of this, it was thought, but not tested, that the vasoconstrictive response might be more uniform and would be less dependent on thermal variations. One potential advantage of the palmar finger site is that because its resting blood flow is usually larger than at the dorsum, the effect of the biological zero is relatively less important. Thus, in a clinical setting, measurement and subsequent subtraction of the biological zero to evaluate the patient's IGR may not always be required. However, this possibility has not been systematically studied.

An estimate of the lower limit of differences between hands of a normal population may be made based on the observed maximum differences obtained in the present normal group (Table 1). Based on a 2 SD limit, the largest difference range is that of the second IGR which yields a lower limit of $28 \cdot 6 \%$. Thus it would seem that bilateral IGR that differ in value by more than $30 \%$ points might be used as a tentative indicator of bilateral asymmetry.

In summary, these results indicate that hand-dominance, in right-handed persons, is not a factor that is likely to significantly effect IGR differentials determined on the finger dorsum of paired-hands.

\section{References}

Abbot NC, Beck JS. Biological zero in laser Doppler measurements in normal, ischaemic and inflamed human skin. Int J Microcirc Clin Exp (1993); 12: 89-98.

Birklein F, Riedl B, Neundorfer B, Handwerker HO. Sympathetic vasoconstrictor reflex pattern in patients with complex regional pain syndrome. Pain (1998); 75: 93-100.

Bolton B, Carmichael EA, Sturup G. Vasoconstriction following deep inspiration. J Physiol (1936); 86: 83-94.

Du Buf-Vereijken PW, Netten PM, Wollersheim H, Festen J, Thien T. Skin vasomotor reflexes during inspiratory gasp: standardization by spirometric control does not improve reproducibility. Int J Microcirc Clin Exp (1997); 17: 86-92.

Hilz MJ, Stemper B, Axelrod FB. Sympathetic skin response differentiates hereditary sensory autonomic neuropathies Iii and IV. Neurology (1999); 52: 1652-1657.

Jakobsson A, Nilsson GE. Prediction of sampling depth and photon pathlength in laser Doppler flowmetry. Med Biol Eng Comput (1993); 31 : 301-307.

Kernick DP, Tooke JE, Shore AC. The biological zero signal in laser Doppler fluximetry - origins and practical implications. Pflugers Arch (1999); 437: 624-631.

Khan F, Spence VA, Wilson SB, Abbot NC. Quantification of sympathetic vascular responses in skin by laser Doppler flowmetry. Int J Microcirc Clin Exp (1991); 10, 145-153.

Khan F, Spence VA, Belch JJ. Cutaneous vascular responses and thermoregulation in relation to age. Clin Sci (Lond) (1992); 82: 521-528.

Lau YT, Liu CF, Tsai CC. Cutaneous vasoconstrictor response induced by inspiratory gasp in relation to sex and age. Clin Sci (Lond) (1995); 89: 233-237.

Littleford RC, Khan F, Belch JJ. Impaired skin vasomotor reflexes in patients with erythromelalgia. Clin Sci (Lond) (1999); 96: 507-512.

Liu CF, See LC, Lau YT. Microcirculatory vasoconstrictor response: relationship with vital capacity and smoking. Life Sci (1996); 59: 1031-1037.

Mayrovitz HN. Assessment of Human Microvascular Function. In: Analysis of Cardiovascular Function (eds Drzewiecki, G, Li, J) (1998), pp. 248-273 Springer, New York.

Mayrovitz HN, Groseclose EE. Inspiration-induced vascular responses in finger dorsum skin. Microvasc Res (2002a); 63: 227-232.

Mayrovitz HN, Groseclose EE. Neurovascular responses to sequential deep inspirations assessed via laser-Doppler perfusion changes in dorsal finger skin. Clin Physiol Funct Imaging (2002b); 22: 4954. 
74 Inspiration-induced vasoconstrictive responses, H. N. Mayrovitz and E. E. Groseclose

Mayrovitz HN, Leedham JA. Laser-Doppler imaging of forearm skin: perfusion features and dependence of the biological zero on heatinduced hyperemia. Microvasc Res (2001); 62: 74-78.

Mueck-Weymann M, Rauh R. Do preceding vasoconstrictions influence the 'inspiratory gasp test'? Clin Physiol Funct Imaging (2002); 22: 206209.

Netten PM, Wollersheim H, Thien T, Lutterman JA. Skin microcirculation of the foot in diabetic neuropathy. Clin Sci (Lond) (1996); 91: 559-565.

Nilsson GE, Tenland T, Oberg PA. Evaluation of a laser Doppler flowmeter for measurement of tissue blood flow. Ieee Trans Biomed Eng (1980); 27: 597-604.
Oberg PA, Tenland T, Nilsson GE. Laser-Doppler flowmetry-a noninvasive and continuous method for blood flow evaluation in microvascular studies. Acta Med Scand Suppl (1984); 687: 17-24.

Oberle J, Elam M, Karlsson T, Wallin BG. Temperature-dependent interaction between vasoconstrictor and vasodilator mechanisms in human skin. Acta Physiol Scand (1988); 132: 459-469.

Rauh R, Posfay A, Muck-Weymann M. Quantification of inspiratoryinduced vasoconstrictive episodes: a comparison of laser Doppler fluxmetry and photoplethysmography. Clin Physiol Funct Imaging (2003); 23: 344-348.

Schurmann M, Gradl G, Furst H. A standardized bedside test for assessment of peripheral sympathetic nervous function using laser Doppler flowmetry. Microvasc Res (1996); 52: 157-170. 